\title{
Efficacy Evaluation Of Albumin-Bound Paclitaxel Combined With Carboplatin As Neoadjuvant Chemotherapy For Primary Epithelial Ovarian Cancer
}

Huan Wang ( $\nabla$ wanghuan@hrbmu.edu.cn )

Harbin Medical University Third Hospital: Tumor Hospital of Harbin Medical University https://orcid.org/0000-0002-3035-1675

\section{Yimin Han}

Harbin Medical University Third Hospital: Tumor Hospital of Harbin Medical University

\section{Research}

Keywords: Albumin-bound paclitaxel combined with carboplatin, neoadjuvant chemotherapy for epithelial ovarian cancer, efficacy evaluation

Posted Date: December 2nd, 2020

DOl: https://doi.org/10.21203/rs.3.rs-117727/v1

License: (c) (i) This work is licensed under a Creative Commons Attribution 4.0 International License.

Read Full License 


\section{Abstract}

Background: This study aimed to compare the efficacy of albumin-bound paclitaxel combined with carboplatin (Nab-TC) with that of traditional solvent-based paclitaxel combined with carboplatin (TC) as a neoadjuvant chemotherapy regimen for primary epithelial ovarian cancer.

Methods: Seventy-six patients with advanced primary epithelial ovarian cancer admitted for treatment at the Third Affiliated Hospital of Harbin Medical University from January 2015 to August 2020 were retrospectively selected. All patients underwent surgery after two courses of neoadjuvant chemotherapy with a combination of paclitaxel and carboplatin. Among the patients included for study, 34 were in the Nab-TC program and 42 in the TC program.

Results: After two courses of chemotherapy, the ORR value was $52.38 \%$ in the TC group and $58.82 \%$ in the Nab-TC group ( $P=0.355)$. The CA125 value of the Nab-TC group decreased by $88.28 \%$ and that of the TC group by $86.99 \%(P=0.358)$. Overall operation time, intraoperative blood loss and postoperative hospital time of patients in the Nab-TC group were lower $(P>0.05)$ and length of total hospital stay was significantly lower $(P<0.05)$ relative to the TC group. Moreover, incidence of bone marrow suppression, abnormal liver function, arrhythmia and hyperglycemia in the Nab-TC group were lower $(P>0.05)$ and total incidence of nausea and vomiting as well as grade III and IV stages were significantly lower $(P<0.05)$ than those in the TC group. However, the frequency of acral numbness in Nab-TC group was higher compared to the TC group $(P<0.05)$.

Conclusion: The efficacy of the Nab-TC regimen as neoadjuvant chemotherapy for advanced primary epithelial ovarian cancer was equivalent to that of the TC regimen, along with a lower incidence of adverse reactions, supporting its therapeutic value in the clinic.

\section{Background}

Ovarian cancer is the leading cause of death from gynecological cancers worldwide. Due to its asymptomatic presentation and lack of effective screening methods, tumors in more than $75 \%$ patients will have spread outside the pelvis region at the time of diagnosis. The main goal for advanced ovarian cancer is satisfactory cytoreductive surgery. In cases where this is not achieved, intermittent debulking surgery (IDS) following neoadjuvant chemotherapy (NACT) has been accepted as an alternative therapeutic strategy for advanced ovarian cancer ${ }^{[1]}$. Earlier studies have disclosed no significant differences between the combined application of NACT with IDS and primary debulking surgery (PDS) in terms of overall or progression-free survival, along with fewer postoperative complications and high rates of surgical elimination ${ }^{[2]}$. Neoadjuvant chemotherapy is considered for patients with stage III to IV cancer presenting large tumors for whom satisfactory cytoreductive surgery is not adequately achieved (residual lesions $\leq 1 \mathrm{~cm}$ ) or those at greater surgical risk ${ }^{[3,4]}$. The National Comprehensive Cancer Network (NCCN) practice guidelines recommend that all intravenous drug regimens for first-line postoperative adjuvant chemotherapy for advanced epithelial ovarian cancer can additionally be used as NACT. Paclitaxel 
combined with carboplatin is considered the standard first-line chemotherapy regimen for advanced ovarian cancer. Taxol drugs are divided into two types, specifically, paclitaxel (solvent, liposome and albumin-bound types) and docetaxel.

Solvent-based paclitaxel, a semi-synthetic drug, is a polyoxyethyl castor oil solvent that forms micelles to wrap paclitaxel. The formulation has relatively poor water solubility and is prone to triggering allergic reactions. Glucocorticoid pretreatment is often thus required before its application ${ }^{[5]}$. Compared with traditional solvent-based paclitaxel, albumin-bound paclitaxel combines hydrophobic paclitaxel and human serum albumin carrier, which decomposes more readily in the body and effectively transports paclitaxel to tumor tissues via endocytosis. Moreover, albumin-bound paclitaxel has a large clinical dose range ( $80-375 \mathrm{mg} / \mathrm{m}^{2}$ ) and the recommended dose for ovarian cancer chemotherapy is $260 \mathrm{mg} / \mathrm{m}^{2}[6]$. Allergic reactions to this formulation are rare and glucocorticoid pretreatment prior to clinical application is not required. The infusion time is short and therefore highly convenient as a clinical regimen. To date, albumin-bound paclitaxel has been employed as neoadjuvant chemotherapy for breast and cervical cancer types. In this study, we have compared the efficacy of Nab-TC and TC regimens as neoadjuvant chemotherapy for epithelial ovarian cancer to ascertain the advantages of albumin binding and establish the utility of type paclitaxel as therapy for ovarian cancer.

\section{Methods}

\section{Inclusion criteria}

Patients with primary epithelial ovarian cancer unable to achieve satisfactory tumor reduction following direct surgery at the Affiliated Tumor Hospital of Harbin Medical University from January 2015 to August 2020 were selected for study. After comprehensive assessment by surgeons and anesthesiologists, patients at higher surgical risk, such as those with advanced age or advanced epithelial ovarian cancer assessed based on the criteria of Eastern cooperative oncology group (ECOG) or American Society of Anesthesiologists (ASA) were selected. Epithelial ovarian cancer types relatively sensitive to chemotherapy (such as high-grade serous adenocarcinoma and poorly differentiated endometrioid adenocarcinoma that could not be evaluated using pathology and imaging examinations (MR/PETCT/enhanced CT));Patients with advanced epithelial ovarian cancer (mesenteric tumor infiltration, extensive involvement of the diaphragm, and multi-segmental intestinal involvement);Patients who could independently complete the questionnaire of this study and voluntarily cooperate with the guidance and arrangements of medical staff; Those with no contraindications to chemotherapy and those who provided signed informed consent were included.

\section{Exclusion criteria}

Patients with other major organ dysfunctions such as intracranial metastases or vertebral body metastases with nerve compression symptoms; Poor coordination as well as those either unwilling to 
follow the instructions and arrangements of medical staff; Allergic to the drugs in this study and pregnant or lactating subjects were excluded.

\section{Basic patient characteristics}

Overall, 34 and 46 patients were included in the Nab-TC and TC groups. Basic demographic and disease status data of the two patient groups were not significantly different $(P>0.05$; Table 1$)$. Prior to chemotherapy, patients from both groups required hematuria, coagulation, liver and kidney function analyses as well as electrocardiography to rule out the possibility of chemotherapy contraindications. All patients received systemic intravenous chemotherapy. Overall, 21 days of treatment were conducted and a total of two courses used. This study was approved by the ethics committee of our hospital and all patients signed an informed consent form.

Table 1

Comparison of characteristics of the two groups

\begin{tabular}{|lllll|}
\hline Characteristic & Nab-TC( $\mathbf{n}=34)$ & TC( $\mathbf{n}=42)$ & Statistics & P value \\
\hline Age (years) & $55.29 \pm 8.185$ & $56.14 \pm 6.381$ & $\mathrm{t}=-0.508$ & 0.613 \\
\hline BMI & $24.40 \pm 4.873$ & $22.69 \pm 3.547$ & $\mathrm{t}=1.77$ & 0.081 \\
\hline FIGO stage & & $\chi^{2}=0.227$ & 0.634 \\
\hline III & $26(76.47 \%)$ & $34(80.95 \%)$ & & \\
\hline IV & $8(23.53 \%)$ & $8(19.05 \%)$ & & \\
\hline Pathological type & & & - & $0.499^{*}$ \\
\hline Serous adenocarcinoma & $34(100.0 \%)$ & $40(95.24 \%)$ & & \\
\hline Endometrioid adenocarcinoma & $0(0.0 \%)$ & $2(4.76 \%)$ & & \\
\hline *Fisher's exact test & & & & \\
\hline
\end{tabular}

\section{Treatment methods}

The therapeutic dose of albumin-bound paclitaxel for the Nab-TC group was $260 \mathrm{mg} / \mathrm{m}^{2}$, and $100 \mathrm{mg}$ was mixed into $20 \mathrm{~mL}$ of $0.9 \%$ sodium chloride injection for preparation with slow instillation for 30 minutes. The patient did not undergo anti-allergy pretreatment and there was no need to monitor vital signs during the infusion process. The traditional solvent-based paclitaxel regimen required desensitization treatment prior to application including oral dexamethasone $7.5 \mathrm{mg}$ at 12 hours before treatment, oral dexamethasone $7.5 \mathrm{mg}$ at 6 hours before treatment, and oral diphenhydramine $50 \mathrm{mg}$ at 30 minutes before treatment. The treatment dose range was $135-175 \mathrm{mg} / \mathrm{m}^{2}$. Overall, $30 \mathrm{mg}$ of traditional paclitaxel was dissolved into $100 \mathrm{~mL}$ of $0.9 \%$ sodium chloride injection and instilled slowly. The patient was observed for the first 30 minutes, and after no abnormal reaction was evident, the remaining drug was dissolved in $500 \mathrm{~mL}$ of $0.9 \%$ sodium chloride for injection. A static point was maintained for 3 hours and vital signs monitored throughout the process. Carboplatin was administered 
intravenously on the second day. The area under the carboplatin curve (AUC) was 5 . The carboplatin dose $(\mathrm{mg})$ was calculated as AUC (usually $5 \mathrm{mg} / \mathrm{mL} / \mathrm{min}) \times$ [creatinine clearance $(\mathrm{mL} / \mathrm{min})+25$ ] and female creatinine clearance rate as $[(140$-age $) \times$ weight $(\mathrm{kg}) \times 1.23] \times 0.85 /$ serum creatinine $(\mu \mathrm{mol} / \mathrm{L})$. To prevent vomiting and nausea during platinum infusion, granisetron hydrochloride $30 \mathrm{mg}$ was intravenously administered prior to chemotherapy. After two courses of treatment, the effects were evaluated.

\section{Tumor Indicators}

After completion of each chemotherapy regimen, the patient underwent assessment for blood tumor index carbohydrate antigen 125 (CA125) as well as gynecological ultrasound or magnetic resonance imaging(MRI) or enhanced computed tomography (CT) examinations. Patients with pleural effusion were subjected to chest CT. Blood was routinely assessed every three days after chemotherapy and liver and kidney function every 7 days. Bone marrow suppression after chemotherapy as well as liver and kidney function damage were evaluated. Pain, peripheral nerve paresthesia, nausea and vomiting were assessed after chemotherapy, along with allergies and other adverse reactions.

\section{Evaluation standards}

The short-term curative effect after two courses of treatment was evaluated according to the solid tumor curative effect evaluation standards (RECIST 1.1 ${ }^{[7]}$. Data were clinically classified into the following disease progression (PD) groups: new lesions appearing with an increased rate of $\geq 20 \%$ : stable disease $(S D)$; tumor enlargement $<20 \%$, tumor reduction $<30 \%$ : partial remission (PR); tumor reduction $\geq 30 \%$ with a duration of more than 4 weeks: complete remission (CR), whereby the mass disappeared completely for more than 4 weeks. The objective remission rate (ORR) was calculated as (PR + CR)/total number of cases $\times 100 \%$, and record the patient's tumor indicators(CA125) change.According to the criteria of WHO for evaluating subacute and acute toxicity of anti-tumor drugs, adverse reactions to chemotherapy were classified as grades I to IV.

\section{Statistical analysis}

SPSS 25.0 software was used for statistical analysis. Measurement data were expressed as mean \pm standard deviation $(\overline{\mathrm{x}} \pm s)$. Counting data is expressed as number of cases and rate (\%).The independent sample $t$-test was used for comparison of continuous variables and chi-square or Fisher's exact test for comparison of categorical variables between groups. Owing to missing values, the mixed-effects model was adopted. Data were considered significant at $\mathrm{P}<0.05$. GraphPad 7.0 was applied to plot data trends.

\section{Results}

Age, body mass index $\left(\mathrm{BMI}=\right.$ weight $(\mathrm{kg}) /$ height $\left.2\left(\mathrm{~m}^{2}\right)\right)$, FIGO staging and pathological type were comparable between the two groups (Table 1).

\section{Comparison of CA125 levels as a malignancy index}


The CA125 value of the Nab-TC group decreased by $88.28 \%$, while that of the TC group declined by $86.99 \%$.Using the mixed-effects model, the interaction effects of time and grouping were not significant within the fixed effect $(F=0.410, P=0.746(>0.05))$, and therefore, only the respective main effects required evaluation. In other words, the difference between the Nab-TC and TC groups at each time-point was not statistically significant $(F=0.850, P=0.358(>0.05))$; Time was significant $(F=17.299, P=0.000$ $(<0.05)$ ), it can be considered that after two courses of chemotherapy, the CA125 value of the two groups decreased significantly compared with before chemotherapy, and the effect was significant. The Bonferroni method was applied for pairwise comparison. Notably, the CA125 value before chemotherapy was significantly higher than that during the three stages after chemotherapy, and the value after the first course chemotherapy was significantly greater than that after the second course of chemotherapy. We observed no significant differences after the course of treatment and after the operation following chemotherapy between the groups (Table 2).

Table 2

Comparison of CA125 values between the two groups $(\bar{X} \pm s)$

\begin{tabular}{|lllll|}
\hline Groups & $\begin{array}{l}\text { Before } \\
\text { chemotherapy }\end{array}$ & $\begin{array}{l}\text { After one course of } \\
\text { chemotherapy }\end{array}$ & $\begin{array}{l}\text { After two courses } \\
\text { of chemotherapy }\end{array}$ & After the operation \\
\hline $\begin{array}{l}\text { Nab-TC } \\
\text { group }\end{array}$ & $3487.38 \pm 5604.045$ & $\begin{array}{l}1204.38 \pm \\
2211.191\end{array}$ & $\begin{array}{l}408.68 \pm \\
1237.448\end{array}$ & $92.81 \pm 169.811$ \\
\hline TC group & $2519.91 \pm 3490.553$ & $\begin{array}{l}1119.25 \pm \\
1846.789\end{array}$ & $327.72 \pm 494.262$ & $140.96 \pm 397.466$ \\
\hline
\end{tabular}

GraphPad7.0 plot data trends.

\section{Comparison of operation indices}

Two independent sample $t$-tests were employed to analyze differences in operation time, blood loss, total hospitalization and postoperative hospitalization days between the two groups. The average operation time was $134.71 \pm 40.805 \mathrm{~min}$ for the Nab-TC group and $134.90 \pm 46.204 \mathrm{~min}$ for the TC group $(\mathrm{t}=-0.02$, $\mathrm{P}=0.984(>0.05))$, which were not considered significantly different. Similarly, no marked differences in blood loss and postoperative hospital stay periods were observed between the two groups $(P>0.05)$. The total length of hospital stay for the Nab-TC group was $10.53 \pm 2.092$ days on average while that for the TC group was recorded as $13.29 \pm 4.363$ days $(t=-3.379, P=0.001(<0.05))$, which was significantly higher (Table 3). 
Table 3

Comparison of surgical indicators $(\overline{\mathrm{x}} \pm \mathrm{s})$

\begin{tabular}{|lllll|}
\hline Surgical index & Nab-TC $(\mathbf{n}=\mathbf{3 4})$ & TC $(\mathbf{n}=\mathbf{4 2})$ & T value & P value \\
\hline Operation time (min) & $134.71 \pm 40.805$ & $134.90 \pm 46.204$ & -0.02 & 0.984 \\
\hline Intraoperative blood loss (mL) & $155.88 \pm 133.578$ & $183.81 \pm 139.003$ & -0.886 & 0.378 \\
\hline Total hospitalization time (day) & $10.53 \pm 2.092$ & $13.29 \pm 4.363$ & -3.379 & 0.001 \\
\hline Postoperative hospital stay (day) & $7.0 \pm 1.875$ & $8.29 \pm 3.591$ & -1.888 & 0.063 \\
\hline
\end{tabular}

\section{Efficacy analysis}

Fisher's chi-square test was applied for analysis of treatment efficacy between the groups. The differences between ORR values of the two groups were not statistically significant $(P=0.355(>0.05)$; Table 4).

Table 4

Analysis of treatment efficacy in the two groups

\begin{tabular}{|llllll|}
\hline Group & CR & PR & SD & PD & ORR \\
\hline Nab-TC Group $(n=34)$ & 0 & 20 & 13 & 1 & $58.82 \%$ \\
\hline TC Group $(n=42)$ & 0 & 22 & 20 & 0 & $52.38 \%$ \\
\hline
\end{tabular}

\section{Adverse reactions}

Due to the relatively small number of cases $(n=76)$, Fisher's precise chi-square analysis was used to evaluate differences in the incidence of adverse reactions among groups. Acral numbness and nausea and vomiting in the two groups were classified as grades I-IV. The incidence of acral numbness was significantly higher in the Nab-TC group while the total incidence of nausea and vomiting (in particular, grades III-IV) were higher in the TC group (Table 5). 
Table 5

Incidence of adverse reactions induced by the two chemotherapeutic regimens ( $\mathrm{n} \%)$

\begin{tabular}{|lllllll|}
\hline Adverse reactions & \multicolumn{2}{l}{ Nab-TC(n=34) (n\%) } & \multicolumn{2}{l|}{ TC(n=42)(n\%) } & $\begin{array}{l}\text { P } \\
\text { value }\end{array}$ & $\begin{array}{l}\text { value } \\
\text { valu }\end{array}$ \\
\cline { 2 - 7 } & I-IV & III-IV & I-IV & III-IV & I-IV & III-IV \\
\hline $\begin{array}{l}\text { Bone marrow } \\
\text { suppression }\end{array}$ & $16(47.05 \%)$ & $4(11.76 \%)$ & $29(69.05 \%)$ & $12(28.57 \%)$ & 0.064 & 0.094 \\
\hline Numbness & $28(82.35 \%)$ & $8(23.53 \%)$ & $22(52.38 \%)$ & $7(16.67 \%)$ & 0.008 & 0.565 \\
\hline Nausea and vomiting & $16(47.05 \%)$ & $4(11.76 \%)$ & $35(83.33 \%)$ & $15(35.71 \%)$ & 0.001 & 0.019 \\
\hline Abnormal liver function & $4(11.76 \%)$ & - & $7(16.67 \%)$ & - & 0.745 & - \\
\hline $\begin{array}{l}\text { Abnormal kidney } \\
\text { function }\end{array}$ & $0(0)$ & - & $0(0)$ & - & - & - \\
\hline Allergic reaction & $0(0)$ & - & $0(0)$ & - & - & - \\
\hline Arrhythmia & $4(11.76 \%)$ & - & $7(16.67 \%)$ & - & 0.745 & - \\
\hline High blood sugar & $2(5.88 \%)$ & - & $6(14.28 \%)$ & - & 0.285 & - \\
\hline Hair loss & $34(100 \%)$ & - & $42(100 \%)$ & - & - & - \\
\hline
\end{tabular}

\section{Discussion}

Here, we evaluated the efficacy of the Nab-TC regimen as neoadjuvant chemotherapy for ovarian cancer. To this end, the tumor shrinkage rate, degree of CA125 decline, ORR value, incidence of adverse reactions, and intraoperative and postoperative indicators were compared between groups treated with Nab-TC and TC regimens. Satisfactory shrinkage was achieved for both groups. Based on tumor shrinkage rate, ORR of the TC group was greater than that of the Nab-TC group, but not to a significant extent. The incidence of nausea and vomiting as well as length of hospital stay of the Nab-TC group were significantly lower relative to the TC group. The finding that the Nab-TC regimen can reduce the occurrence of adverse reactions and length of hospitalization along with promoting effective tumor shrinkage supports its clinical utility in the treatment of primary epithelial ovarian cancer.

A study by Mahdi et al. ${ }^{[8]}$ showed that at least $90 \%$ decrease in CA125 level after NACT is related to more complete IDS and reduced incidence of bowel resection but does not improve survival results. In our investigation, the CA125 value of the Nab-TC group decreased by $88.28 \%$ and that of the TC group by $86.99 \%$. The baseline level of CA125 in the Nab-TC group before chemotherapy was higher, but no significant differences were observed between the two groups (Table 2). Satisfactory outcomes with cytoreductive surgery were achieved for both patient groups. Earlier research by Rodriguez et al. ${ }^{[9]}$ demonstrated that patients who undergo NACT-IDS achieve a high rate of optimal cytoreduction and after treatment with taxane and platinum-based chemotherapy, patients with preoperative CA125 levels less 
than $100 \mathrm{U} / \mathrm{mL}$ were highly likely to be cytoreduced to no residual disease. Moreover, subjects with preoperative $\mathrm{CA} 125$ values $\leq 230 \mathrm{U} / \mathrm{mL}$ are reported to have better progression-free survival ${ }^{[10]}$. The proportion of patients with CA 125 values $>230 \mathrm{U} / \mathrm{mL}$ before chemotherapy was $94.12 \%$ in the Nab-TC group and $80 \%$ in the TC group. The proportions of patients showing reduction of CA125 values to below $230 \mathrm{U} / \mathrm{mL}$ and $100 \mathrm{U} / \mathrm{mL}$ after two courses of chemotherapy were $75 \%$ and $68.75 \%$ in the Nab-TC group and $66.67 \%$ and $8.33 \%$ in the TC group, respectively (data not shown). Accordingly, we concluded that Nab-TC and TC programs involving the same neoadjuvant chemotherapy regimens had similar efficacy. The issue of whether there is a significant difference in the five-year and overall survival rates requires further validation.

In clinical guidelines of NACT usage for advanced epithelial ovarian cancer, platinum in combination with taxanes is recommended. A combination of paclitaxel and carboplatin chemotherapy is considered the standard treatment for women with advanced ovarian cancer. Paclitaxel is an anti-microtubule drug that achieves tumor suppressor effects by interfering with cell mitosis. However, due to its killing effect on normal cells and toxic side effects, its core therapeutic value is weakened. Albumin-bound paclitaxel is a novel type of chemotherapeutic drug generated by combining paclitaxel with albumin. The drug formulation has good solubility and antihistamine treatment is not required before administration, which simplifies the treatment process and achieves optimal therapeutic effects ${ }^{[11]}$. Nabholtz and coworkers $^{[12]}$ reported that at a single dose of ordinary paclitaxel of $135-175 \mathrm{mg} \cdot \mathrm{m}^{2}$, the incidence of sensory neurotoxicity was $46-70 \%$ and that of sensory neurotoxicity to degree 3 or 4 was $3-7 \%$. This finding may be related to the demyelination and neuronal degeneration caused by organic solvents and polyoxyethylene castor oil. At an increased dose of paclitaxel to $200-250 \mathrm{mg} \cdot \mathrm{m}^{2}$, the incidence of degree 4 sensory neurotoxicity was determined as $9-12 \%$. In this study, the incidence of acral numbness was higher in the Nab-TC group, which could be attributable to the larger dose. However, patients in the NabTC group were less likely to experience common side-effects, such as bone marrow suppression, liver and kidney abnormalities, arrhythmia, nausea and vomiting. Treatment with albumin-bound paclitaxel does not require glucocorticoid pretreatment or trigger allergic reactions before clinical administration, and reduces the incidence of high blood sugar caused by long-term oral glucocorticoids. Furthermore, the clinical infusion time is short, which improves patient quality of life and chemotherapy tolerance.

\section{Conclusions}

This retrospective study showed that after two courses of neoadjuvant chemotherapy for primary epithelial ovarian cancer, ORR of the Nab-TC group was slightly higher than that of the TC group $(P>$ 0.05 ) and both groups achieved satisfactory tumor cell reduction. No postoperative infection and pulmonary embolism occurred. The operation time and intraoperative blood loss in the Nab-TC group ( $\mathrm{P}$ $>0.05)$ as well as length of hospital stay $(P<0.05)$ were lower than those recorded in the TC group. Moreover, the incidence of bone marrow suppression, liver and kidney abnormalities, arrhythmia, and hyperglycemia in the Nab-TC group $(P>0.05)$ and total incidence of nausea and vomiting and grade III and IV were lower than those in the TC group $(P<05)$ while the incidence of numbness was higher $(P<$ 
0.05). In summary, for patients with primary epithelial ovarian cancer, the clinical efficacy of Nab-TC was comparable to that of the traditional TC regimen, along with reduced incidence of adverse reactions and short infusion times, supporting its therapeutic utility and further development as neoadjuvant therapy.

\section{Limitations And Deficiencies}

Our findings were limited by the small sample size and lack of long-term follow-up. Further prospective and randomized controlled trials with larger sample sizes and long-term analyses are warranted.

Furthermore, relevant studies on postoperative chemotherapy resistance and follow-up of progressionfree and overall survival times of patients were lacking.

\section{Abbreviations}

Nab-TC, albumin-bound paclitaxel combined with carboplatin

TC, solvent-based paclitaxel combined with carboplatin

NACT, neoadjuvant chemotherapy

IDS, intermittent cytoreductive surgery

PDS, primary debulking surgery

NCCN, National Comprehensive Cancer Network

ECOG, Eastern cooperative oncology group

ASA, American Society of Anesthesiology

BMI, Body Mass Index

CA125, Carbohydrate Antigen 125

\section{Declarations}

\section{Ethics approval and consent to participate}

This study was conducted in accordance with Helsinki Declaration II and approved by the Institutional Review Boards of Harbin Medical University.

\section{Consent for publication}

Not applicable. 
Based on approval by the ethics committee, medical records in the hospital could be used for scientific studies, but sharing of data was not allowed.

\section{Competing interests}

The authors have no competing interests to declare.

\section{Funding}

Unfunded.

\section{Author contributions}

$\mathrm{YH}$ contributed to the conception and design of the study. HW and $\mathrm{YH}$ acquired the data. HW conducted literature searches. HW analyzed and interpreted the data. HW drafted and revised the article. YH provided the final approval of the version to be submitted for publication. All authors read and approved the final manuscript.

\section{Acknowledgements}

We are grateful to Pi Tie (Department of Statistics, Chongqing Medical University) for contributing to statistical analyses and Lingyun Fan (The Third Affiliated Hospital of Harbin Medical University) for help with data searches.

We thank International Science Editing ( http://www.internationalscienceediting.com ) for editing this manuscript.

\section{Author details}

[1]Department of Gynaecology, The Third Affiliated Hospital of Harbin Medical University, No.150, Haping Road, Harbin 150081, China.

\section{References}

1. Lecointre $L$, Velten $M$, Lodi $M$, et al. Impact of neoadjuvant chemotherapy cycles on survival of patients with advanced ovarian cancer: A French national multicenter study (FRANCOGYN)[J]. Eur J Obstet Gynecol Reprod Biol, 2020,245:64-72.

2. Kehoe S, Hook J, Nankivell M, et al. Primary chemotherapy versus primary surgery for newly diagnosed advanced ovarian cancer (CHORUS): An open-label, randomised, controlled, non-inferiority trial[J]. Lancet, 2015,386(9990):249-257.

3. Qin $M$, Jin $Y, M a ~ L$, et al. The role of neoadjuvant chemotherapy followed by interval debulking surgery in advanced ovarian cancer: A systematic review and meta-analysis of randomized controlled trials and observational studies[J]. Oncotarget, 2018,9(9):8614-8628. 
4. Wright A A, Bohlke K, Armstrong D K, et al. Neoadjuvant chemotherapy for newly diagnosed, advanced ovarian cancer: Society of Gynecologic Oncology and American Society of Clinical Oncology Clinical Practice Guideline[J]. J Clin Oncol, 2016,34(28):3460-3473.

5. Fader A N, Rose P G. Abraxane for the treatment of gynecologic cancer patients with severe hypersensitivity reactions to paclitaxel[J]. Int J Gynecol Cancer, 2009,19(7):1281-1283.

6. Teng X Y, Guan Z Z, Yao Z W, et al. [A tolerability study of A cremophor-free albumin bound nanoparticle paclitaxel intravenously administered in patients with advanced solid tumor][J]. Ai Zheng, 2004,23(11 Suppl):1431-1436.

7. Nishino M, Jagannathan J P, Ramaiya N H, et al. Revised RECIST guideline version 1.1: What oncologists want to know and what radiologists need to know[J]. AJR Am J Roentgenol, 2010,195(2):281-289.

8. Mahdi H, Maurer K A, Nutter B, et al. The impact of percent reduction in CA-125 levels on prediction of the extent of interval cytoreduction and outcome in patients with advanced-stage cancer of Mullerian origin treated with neoadjuvant chemotherapy[J]. Int J Gynecol Cancer, 2015,25(5):823-829.

9. Rodriguez N, Rauh-Hain J A, Shoni M, et al. Changes in serum CA-125 can predict optimal cytoreduction to no gross residual disease in patients with advanced stage ovarian cancer treated with neoadjuvant chemotherapy[J]. Gynecol Oncol, 2012,125(2):362-366.

10. Riedinger J M, Wafflart J, Ricolleau G, et al. CA 125 half-life and CA 125 nadir during induction chemotherapy are independent predictors of epithelial ovarian cancer outcome: results of a French multicentric study[J]. Ann Oncol, 2006,17(8):1234-1238.

11. Saxena A, Schneider B J, Christos P J, et al. Treatment of recurrent and platinum-refractory stage IV non-small cell lung cancer with nanoparticle albumin-bound paclitaxel (nab-paclitaxel) as a single agent[J]. Med Oncol, 2016,33(2):13.

12. Nabholtz J M, Gelmon K, Bontenbal M, et al. Multicenter, randomized comparative study of two doses of paclitaxel in patients with metastatic breast cancer[J]. J Clin Oncol, 1996,14(6):1858-1867.

\section{Figures}




\section{Tendency chart}

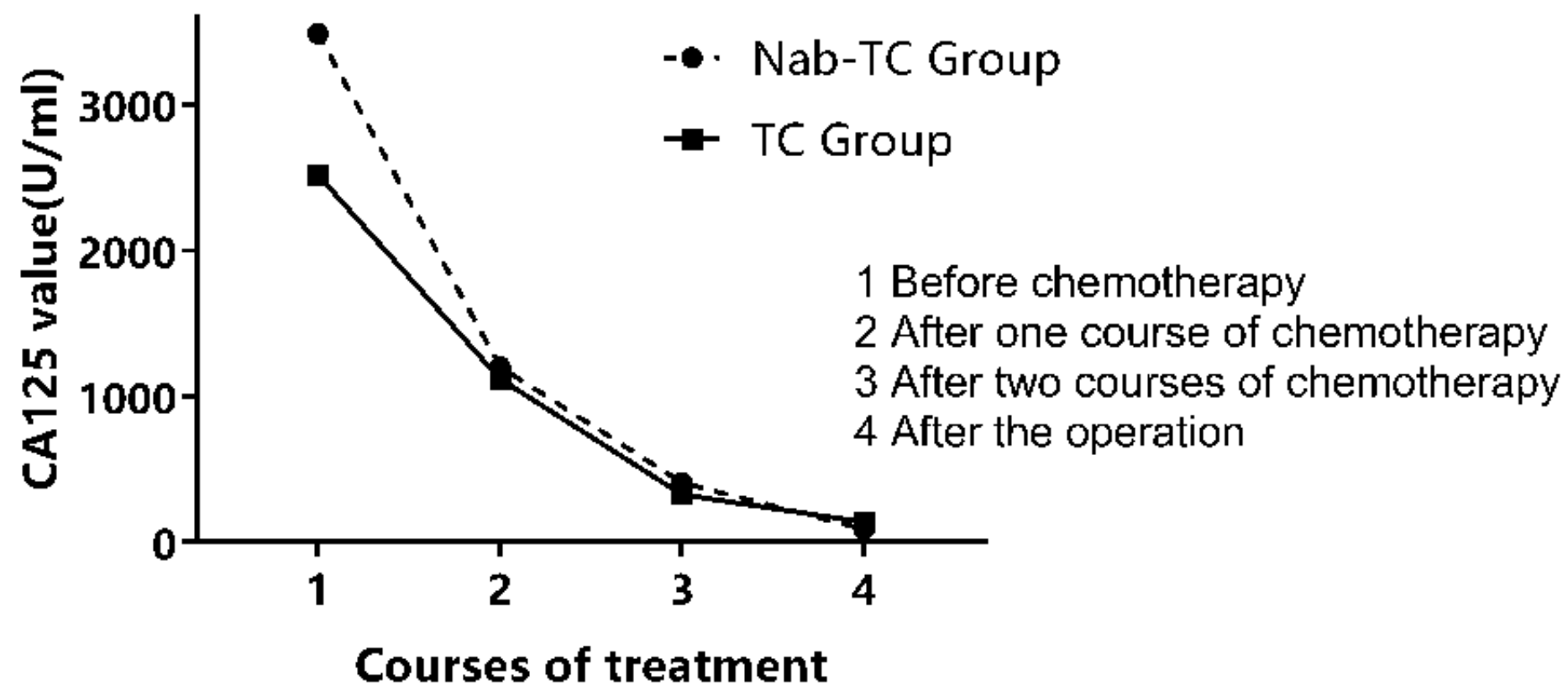

Figure 1

GraphPad7.0 plot data trends. 